\title{
ON THE PROPAGATION OF IONIZATION WAVES
}

\author{
R. Munt and R. S. B. ONG \\ Department of Aerospace Engineering, The University of Michigan, Ann Arbor, Mich. 48104, \\ U.S.A. \\ and \\ D. L. TuRCotTe \\ Graduate School of Aerospace Engineering, Cornell University, Ithaca, N.Y. 14850, U.S.A.
}

(Received 31 January 1969 and in final form 19 March 1969)

\begin{abstract}
A non-linear analysis of ionization wave fronts is given including energy effects. The necessary external energy source is Joule heating due to an applied transverse electric field. The front propagates at a speed which is proportional to the product of the electron sound speed and the square root of the ratio of the electron collision time to the reaction time. The structure of the ionization wave is also obtained.
\end{abstract}

\section{INTRODUCTION}

AN IONIZATION wave may propagate through an initially nonionized gas in much the same way that a flame front propagates through a combustible gas mixture. A continuum theory for the structure of ionization wave fronts has been given by TURCOTTE and ONG (1968). Their theory was based upon a balance between the production and recombination of electrons and ions, the convection of electrons and ions, and the diffusion of electrons. Energy effects were neglected and a constant electron temperature was assumed. A lower bound for the propagation speed of the ionization wave was found.

The purpose of this paper is to extend the analysis of TURCOTTE and Oivg (1968) to include energy effects. A steady plane wave analysis is used in which all quantities are functions only of one spacial coordinate. The electron temperature is assumed to be large compared with the ion and neutral temperatures. The energy source necessary to provide the hot electrons is the Joule heating due to a constant applied transverse electric field. It is also assumed that the background neutral gas is not affected by the ionization front. The problem is solved in the limit of large electrical interaction between the electrons and ions-the ambipolar limit-since this limit is appropriate for most cases of interest. In this limit a single propagation speed is obtained and the structure of the ionization wave is completely determined.

\section{FORMULATION OF THE PROBLEM}

The problem is formulated using a reference frame in which the wave is at rest. A time independent solution is sought which connects the uniform conditions upstream and downstream. The speed of the gas incident on the front is then the propagation speed of the wave. Far upstream the gas is not ionized. We assume that the degree of ionization in the wave and downstream is sufficiently small so that collisions of charged particles with neutral particles dominate over collisions between charged particles in determining the transport coefficients and the neutral gas is not affected either by the ionization process or by the presence of the charged species. Therefore we neglect variations in the mass average velocity $u_{w}$, the neutral gas number density $n_{n}$, and the neutral gas temperature $T$. The ion temperature $T_{i}$ is assumed to be everywhere equal to the neutral gas temperature $T$, and is therefore a constant. 
The electron temperature $T_{e}(x)$ is assumed to be large compared with the neutral gas temperature; consequently the neutrals are ionized only by collisions with the hot electrons and the dominant ionization and recombination processes are given by

$$
A+e \rightleftharpoons A^{+}+e+e
$$

Further details of the model have been given by TURCotTE and ONG (1968). Neglecting ion diffusion the equations for conservation of electrons and ions are (BURGERS, 1960)

$$
\begin{aligned}
& -u_{w} \frac{\mathrm{d} n_{e}}{\mathrm{~d} x}=\frac{\mathrm{d}}{\mathrm{d} x}\left(D_{e} \frac{\mathrm{d} n_{e}}{\mathrm{~d} x}+\frac{8}{13} \frac{D_{e} n_{e}}{T_{e}} \frac{\mathrm{d} T_{e}}{\mathrm{~d} x}+\frac{D_{e} e n_{e}}{k T_{e}} E_{x}\right)+K_{I} n_{n} n_{e}-K_{R} n_{e}^{2} n_{i} \\
& -u_{w} \frac{\mathrm{d} n_{i}}{\mathrm{~d} x}=K_{I} n_{n} n_{e}-K_{R} n_{e}{ }^{2} n_{i} .
\end{aligned}
$$

The ionization reaction is endothermic and the necessary external energy source is the Joule heating which arises from the current flow in an applied transverse electric field. The energy conservation equation for the electrons is

$$
\begin{aligned}
-u_{w} \frac{\mathrm{d}}{\mathrm{d} x}\left(\frac{3}{2} n_{e} k T_{e}\right)= & \frac{\mathrm{d}}{\mathrm{d} x}\left(\frac{40}{13} D_{e} n_{e} k \frac{\mathrm{d} T_{e}}{\mathrm{~d} x}+\frac{55}{26} D_{e} k T_{e} \frac{\mathrm{d} n_{e}}{\mathrm{~d} x}\right. \\
+ & \left.\frac{55}{26} n_{e} e D_{e} E_{x}\right)-3 \frac{n_{e} k^{2} T_{e}}{D_{e} m_{n}}\left(T_{e}-T\right) \\
& -e V_{i}\left(K_{I} n_{n} n_{e}-K_{R} n_{e}{ }^{2} n_{i}\right)+\frac{n_{e} e^{2} D_{e}}{k T_{e}} E_{y}{ }^{2}
\end{aligned}
$$

where $K_{I}$ and $K_{R}$ are the ionization and recombination rate coefficients respectively. These coefficients are functions only of the electron temperature $T_{e}(x)$. The electron diffusion coefficient $D_{e}$ depends on the electron temperature and the neutral gas number density but the latter is a constant. The numerical coefficients in (2), (3) and (4) follow from the hard sphere collision model assumed for the electron-neutral interactions. To complete the set we also require Poisson's equation

$$
\frac{\mathrm{d} E_{x}}{\mathrm{~d} x}=\frac{e}{\varepsilon_{0}}\left(n_{i}-n_{e}\right) .
$$

Upstream the required boundary conditions are $n_{e}=n_{i}=0$, downstream $n_{e}=n_{i}=$ $n_{e 0}$. The electric field component $E_{x}$ vanishes far downstream since the electrical conductivity is finite there and no electric current in the $x$-direction is allowed, so $E_{x}=0$ as $x \rightarrow-\infty$. Upstream the electric field may be finite since the electrical conductivity is zero and therefore a finite value for the electric field does not give an electric current. For a true semi-infinite, one-dimensional problem the finite upstream electric field implies an infinite potential. However for any experiment with a tube of finite length or for a three-dimensional problem the required potential is finite. The solution we obtain shows that the required upstream electric field is directly related to the applied transverse electric field.

For any value of the electron temperature there is a corresponding quasi-equilibrium given by

$$
0=K_{I} n_{e q} n_{n}-K_{R} n_{e q}{ }^{3}
$$


where the subscript $q$ denotes the equilibrium condition at the temperature $T_{e}$ where $n_{e q}\left(T_{e}\right)=n_{i g}\left(T_{e}\right)$. We may also relate the constant transverse component of the electric field, $E_{y}$, to the downstream value of the electron temperature $T_{e 0}$ by

$$
E_{y}{ }^{2}=3 \frac{k^{3} T_{e 0}^{2}}{D_{e 0}^{2} e^{2} m_{n}}\left(T_{e 0}-T\right)
$$

Since $T$ and $E_{y}$ are assumed to be given and constant the downstream electron temperature is determined. Equation (7) is a balance between the energy input to the electrons due to Joule heating and the energy loss through elastic collisions with the neutral gas. However the corresponding increase of the neutral gas temperature is assumed to be negligible since the degree of ionization is small and this occurs on a time scale far greater than that of the reaction in the wave. Substitution of (6) and (7) into (2), (3) and (4) gives

$$
\begin{aligned}
&-u_{w} \frac{\mathrm{d} n_{e}}{\mathrm{~d} x}=\frac{\mathrm{d}}{\mathrm{d} x}\left(D_{e} \frac{\mathrm{d} n_{e}}{\mathrm{~d} x}+\frac{8}{13} \frac{D_{e} n_{e}}{T e} \frac{\mathrm{d} T_{e}}{\mathrm{~d} x}+\frac{D_{e} n_{e} e}{k T_{e}} E_{x}\right)+K_{R} n_{e}\left(n_{e q}{ }^{2}-n_{e} n_{i}\right) \\
&-u_{w} \frac{\mathrm{d} n_{i}}{\mathrm{~d} x}=K_{R} n_{e}\left(n_{e q}{ }^{2}-n_{e} n_{i}\right) \\
&-u_{w} \frac{\mathrm{d}}{\mathrm{d} x}\left(\frac{3}{2} n_{e} k T_{e}\right)= \frac{\mathrm{d}}{\mathrm{d} x}\left(\frac{40}{13} D_{e} n_{e} k \frac{\mathrm{d} T_{e}}{\mathrm{~d} x}+\frac{55}{26} D_{e} k T_{e} \frac{\mathrm{d} n_{e}}{\mathrm{~d} x}+\frac{55}{26} e n_{e} D_{e} E_{x}\right) \\
&-e V_{i} K_{R} n_{e}\left(n_{e q}{ }^{2}-n_{e} n_{i}\right)+ 3 \frac{n_{e} k^{2} T_{e 0}}{D_{e 0} m_{n}}\left[\frac{T_{e 0} D_{e}}{T_{e} D_{e 0}}\left(1-\frac{T}{T_{e 0}}\right)-\frac{T_{e} D_{e 0}}{T_{e 0} D_{e}}\left(\frac{T_{e}}{T_{e 0}}-\frac{T}{T_{e 0}}\right)\right] .
\end{aligned}
$$

We now introduce the following dimensionless variables

$$
\begin{gathered}
M=\frac{n_{e}}{n_{e 0}}, \quad \quad N=\frac{n_{i}}{n_{e 0}}, \quad G=\frac{\varepsilon_{0} u_{w} E_{x}}{e n_{e 0} D_{e 0}}, \\
H=\frac{T_{e}}{T_{e 0}}, \quad \xi=\frac{u_{w}}{D_{e 0}} \int_{0}^{x} \frac{T_{e}}{T_{e 0}} \mathrm{~d} x
\end{gathered}
$$

and for simplicity we assume that

$$
\frac{D_{e}}{D_{e 0}}=\frac{T_{e}}{T_{e 0}}, \frac{K_{R}}{K_{R 0}}=\frac{T_{e 0}}{T_{e}}, \frac{n_{e q}{ }^{2}}{n_{e 0}{ }^{2}}=\exp \left[-\left(\frac{e V_{i}}{k T_{e}}-\frac{e V_{i}}{k T_{e 0}}\right)\right] .
$$

The last relation in (12) follows from the Saha equation. Substitution of (11) and (12) into (5), (8), (9) and (10) gives

$$
\begin{gathered}
\frac{\mathrm{d} M}{\mathrm{~d} \xi}+\frac{\mathrm{d}}{\mathrm{d} \xi}\left(\frac{\mathrm{d} M}{\mathrm{~d} \xi}+\frac{8}{13} \frac{M}{H} \frac{\mathrm{d} H}{\mathrm{~d} \xi}+\beta \lambda M G\right) \\
\quad+\lambda M\left(\exp \left[-\varepsilon\left(H^{-1}-1\right)\right]-M N\right)=0 \\
\frac{\mathrm{d} N}{\mathrm{~d} \xi}+\lambda M\left(\exp \left[-\varepsilon\left(H^{-1}-1\right)\right]-M N\right)=0 \\
\frac{\mathrm{d} G}{\mathrm{~d} \xi}=H(N-M) \\
\frac{\mathrm{d}}{\mathrm{d} \xi}\left(\frac{3}{2} M H\right)+\frac{\mathrm{d}}{\mathrm{d} \xi}\left(\frac{40}{13} M \frac{\mathrm{d} H}{\mathrm{~d} \xi}+\frac{55}{26} H \frac{\mathrm{d} M}{\mathrm{~d} \xi}+\frac{55}{26} \beta \lambda H M G\right) \\
-\varepsilon \lambda M\left(\exp \left[-\varepsilon\left(H^{-1}-1\right)\right]-M N\right)+3 \psi \lambda M H(1-H)=0
\end{gathered}
$$


where

$$
\begin{array}{ll}
\beta=\frac{e^{2} D_{e 0}}{\varepsilon_{0} n_{e 0} k T_{e 0} K_{R 0}}, & \lambda=\frac{K_{R 0} n_{e 0}{ }^{2} D_{e 0}}{u_{v v}{ }^{2}} \\
\psi=\frac{k T_{e 0}}{D_{e 0} K_{R 0} n_{e 0}{ }^{2} m_{n}}, & \varepsilon=\frac{e V_{i}}{k T_{e 0}}, \quad t=\frac{T_{e 0}}{T} .
\end{array}
$$

The required boundary conditions are

$$
\begin{aligned}
& \text { at } \xi \rightarrow+\infty: M=N=0 ; G=G_{0} ; H=H_{0} \\
& \text { at } \xi \rightarrow-\infty: M=N=1 ; G=0 ; H=1
\end{aligned}
$$

where $G_{0}$ and $H_{0}$ are finite unknown constants.

The dimensionless parameter $\beta$ is a measure of the electrical interaction between the ions and electrons. It is easily shown that

$$
\beta \sim\left(\frac{\tau_{\text {reac }}}{\tau_{c}}\right)\left(\frac{L_{c}}{L_{D}}\right)^{2}
$$

where $L_{c}$ is the mean free path for electrons, $L_{D}$ is the Debye length, $\tau_{c}$ is the collision time for electrons, and $\tau_{\text {reac }}$ is the reaction time; all quantities based on $n_{e 0}$. The ratio $\tau_{\text {reac }} / \tau_{c}$ is the average number of electron-neutral collisions required for an ionizing collision. For the range of electron temperatures in which the present analysis is valid $\tau_{\text {reac }} / \tau_{c} \gg 1$. If $\beta \ll 1$, the Debye length is large and the ion and electron number densities may be appreciably different. If $\beta \gg 1$, space charge effects dominate and the number densities of the ions and electrons are neariy equal throughout most of the front. For most applications $\beta$ is a large number.

The dimensionless parameter $\lambda$ is an eigenvalue; it gives the allowed propagation speeds of the ionization front. The dimensionless parameter $\varepsilon$ represents the ratio of the ionization potential to the electron thermal energy and $\exp (-\varepsilon)$ is the fraction of electrons in a Maxwellian distribution with sufficient energy to ionize a neutral. For the required low degree of ionization $\varepsilon$ must be large compared with unity.

The dimensionless parameter $\psi$ is a measure of the energy exchange between the electrons and neutrals. It is easily shown that

$$
\psi \sim\left(\frac{\tau_{\text {reac }}}{\tau_{c}}\right) \frac{m_{e}}{m_{n}} .
$$

We may also view $\psi$ as the ratio of the energy exchange between electrons and neutrals through elastic collisions to that through inelastic ionizing collisions; if $\psi \gg 1$ the electrons within the wave front lose energy predominantly through elastic collisions with the neutrals. Typical values of $\beta$ and $\psi$ are given in the section on numerical calculations. If $\psi$ is large we expect that $T_{e}(x)$ is only slightly lower than $T_{e 0}$.

We combine (13) and (14) and integrate once using the upstream boundary conditions to give

$$
\frac{\mathrm{d} M}{\mathrm{~d} \xi}+\frac{8}{13} \frac{M}{H} \frac{\mathrm{d} H}{\mathrm{~d} \xi}+\beta \lambda M G+M-N=0
$$


Introducing

$$
P=\frac{\mathrm{d} H}{\mathrm{~d} \xi}
$$

we transform (14)-(18) into a set of equations appropriate for a phase space analysis by eliminating the spatial coordinate $\xi$.

$$
\begin{aligned}
& \frac{\mathrm{d} H}{\mathrm{~d} N}=-\frac{P}{\lambda M\left(\exp \left[-\varepsilon\left(H^{-1}-1\right)\right]-M N\right)} \\
& \frac{\mathrm{d} M}{\mathrm{~d} N}=\frac{\frac{8}{13} H^{-1} M P+\beta \lambda M G+M-N}{\lambda M\left(\exp \left[-\varepsilon\left(H^{-1}-1\right)\right]-M N\right)} \\
& \frac{\mathrm{d} G}{\mathrm{~d} N}=\frac{H(M-N)}{\lambda M\left(\exp \left[-\varepsilon\left(H^{-1}-1\right)\right]-M N\right)} \\
& \frac{300}{169} M \frac{\mathrm{d} P}{\mathrm{~d} N}+\left(-\frac{8}{13} H+\frac{300}{169} P\right) \frac{\mathrm{d} M}{\mathrm{~d} N}+\frac{55}{26} H+\varepsilon \\
& -\frac{\frac{3}{2} P+3 \psi \lambda H(1-H)+\frac{55}{26} M P(N-M)}{\lambda M\left(\exp \left[-\varepsilon\left(H^{T-1}-1\right)\right]-M N\right)}
\end{aligned}
$$

The required boundary conditions are

$$
\begin{aligned}
& \text { at } N=0: M=0, G=G_{0}, H=H_{0} \\
& \text { at } N=1: M=1, G=0, H=1 .
\end{aligned}
$$

It is easily shown that the downstream singularity at $N=1$ is a saddle point and the upstream singularity at $N=0$ is a node.

\section{SOLUTION FOR $H_{0}, G_{0}$, AND THE EIGENVALUE $\lambda$}

We expand the variables in a power series about the upstream singularity as follows

$$
\begin{gathered}
H=H_{0}+A N+O\left(N^{2}\right) ; \quad P=B N+O\left(N^{2}\right) \\
M=C N+E N^{2}+O\left(N^{3}\right) ; \quad G=G_{0}+D N+O\left(N^{2}\right) .
\end{gathered}
$$

For convenience we define

$$
\delta=\exp \left(-\varepsilon\left[H_{0}^{-1}-1\right]\right)
$$


Substituting (24) into (20)-(23) and equating the coefficients of like powers of $N$, we obtain

$$
\begin{gathered}
A \lambda C \delta=-B \\
C^{2} \lambda \delta=\beta \lambda G_{0} C+C-1 \\
2 E \lambda C \delta=\beta \lambda C D+E C^{-1}+\frac{8}{13} C B H_{0}^{-1}-\left(\beta \lambda G_{0} C+C-1\right) \\
D \lambda C \delta=H_{0}(C-1) \\
\frac{3}{2} H_{0} C-\frac{55}{26} H_{0}(C-1)+\varepsilon=3 \delta^{-1} H_{0}\left(1-H_{0}\right) \\
\frac{600}{169} B C=\frac{16}{13} H_{0} E+\frac{8}{13} A C+\frac{55}{26} \frac{B(1-C)}{\lambda \delta C}+\frac{3 B+6 \lambda \psi A\left(1-2 H_{0}\right)}{2 \lambda \delta} \\
-\frac{3 \psi \varepsilon\left(1-H_{0}\right) A}{H_{0} \delta}-\frac{55}{26} A .
\end{gathered}
$$

Equations (25)-(30) constitute a set of six equations for the seven unknowns $A, B, C$, $D, E, G_{0}$ and $H_{0}$. However, as noted earlier, $\beta \gg 1$ for most cases of interest. It can be shown (TURCOTTE and ONG, 1968) that in this ambipolar limit the following expansion is valid throughout the front

$$
M=N+O\left(\beta^{-1}\right)
$$

And for large $\beta$ this implies from (24) that

$$
C=1 \text {. }
$$

Substituting this result into (25)-(30) yields

$$
\begin{gathered}
\lambda \delta A=-B \\
\delta=\beta G_{0} \\
(2 \lambda \delta-1) E=\frac{8}{13} B H_{0}^{-1}-\beta \lambda G_{0} \varepsilon A H_{0}^{-2} \\
D=0 \\
\frac{3}{2} H_{0}+\varepsilon=3 \psi H_{0}\left(1-H_{0}\right) \delta^{-1} \\
\frac{600}{169} B=\frac{16}{13} H_{0} E+\frac{8}{13} A+\frac{3}{2} B \lambda^{-1} \delta^{-1}+3 \psi A \delta^{-1}\left(1-2 H_{0}\right) .
\end{gathered}
$$

Equations (33)-(38) constitute a set of six equations in six unknowns. Equation (37) gives $H_{0}$ immediately as a function of $\varepsilon$ and $\psi$ which are known. Then $G_{0}$ may be found from (34). Thus the set of equations (33)-(38) is essentially reduced to the three homogeneous equations (33), (35) and (38) in the three unknowns $A, B$ and $E$. In order that a nontrivial solution exist, the determinant of the coefficients must be equal to zero. This yields

$$
\frac{600}{169} \lambda \delta^{2}=\frac{16}{13} \frac{\left(\frac{8}{13}+\varepsilon H_{0}^{-1}\right)}{(2 \lambda \delta-1)} \lambda \delta^{2}+3 \delta-3 \psi\left(1-2 H_{0}\right)+3 \psi \varepsilon \frac{\left(1-H_{0}\right)}{H_{0}} .
$$


Solutions of (39) give the eigenvalue. Since (39) is quadratic in $\lambda$, it yields two values of $\lambda$, namely $\lambda_{1}$, and $\lambda_{2}$ which depend upon $\varepsilon$ and $\psi$. Note that $H_{0}$ is a function of $\varepsilon$ and $\psi$ as expressed by (37). In Fig. 1 we show the behaviour of $\lambda$ as a function of $\psi$ for a typical value of $\varepsilon$.

From (37) it can be seen by inspection that $H_{0}<1$ for all positive values of $\varepsilon$ and $\psi$. This indicates that the electron temperature decreases upstream. This is reasonable

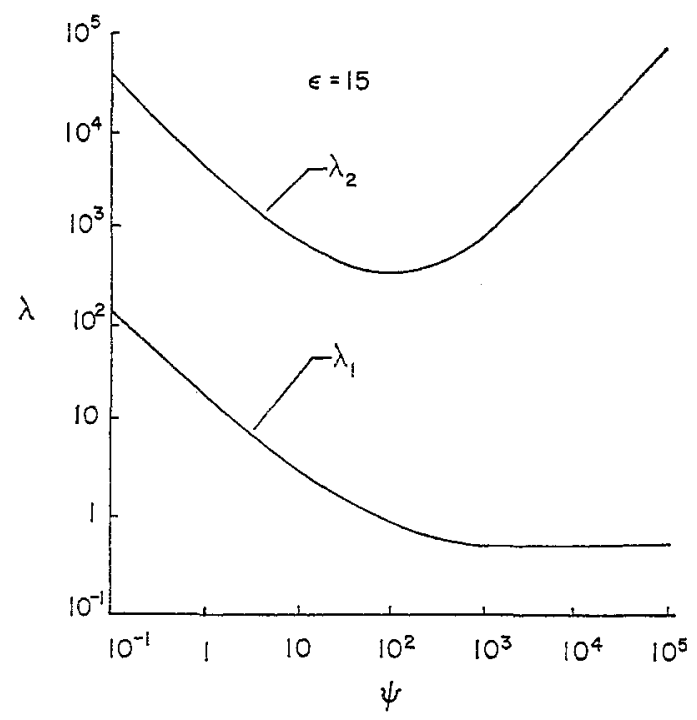

FIG. 1.-Dependence of the eigenvalue $\lambda$ on $\psi$ for $\varepsilon=15$ and $\beta \gg 1$ from (39).

since the ionization reaction is endothermic and thus absorbs heat from the electron gas. Furthermore with $H_{0}<1$ we have $\delta<1$, and hence from (34) we see that

$$
G_{0} \sim O\left(\beta^{-1}\right)
$$

For the case $\psi \gg 1$ it can be shown by numerical calculations that $H_{0}=1+O\left(\psi^{-1}\right)$. This leads to $\delta=1+O\left(\psi^{-1}\right)$. Thus for large values of $\psi$ we may estimate the eigenvalue $\lambda$ as follows. Let

$$
H_{0}=1+\psi^{-1} h_{1}+O\left(\psi^{-2}\right) .
$$

Substituting this into (37) gives

and it follows that

$$
h_{1}=-\left(\frac{\varepsilon}{3}+\frac{1}{2}\right)
$$

$$
H_{0}=1-\psi^{-1}\left(\frac{\varepsilon}{3}+\frac{1}{2}\right)+O\left(\psi^{-2}\right) .
$$

We now substitute (42) into (39) and obtain the following expressions for $\lambda$

$$
\begin{aligned}
& \lambda_{1}=\frac{1}{2}+O\left(\psi^{-1}\right) \\
& \lambda_{2}=\frac{169}{200} \psi+O(1) .
\end{aligned}
$$


It was noted previously that large values of $\psi$ imply a near constant $T_{e}$ throughout the wave. Hence in the limit as $\psi \rightarrow \infty$ it is appropriate to replace the electron energy equation with the condition $H=1$. If we solve for $\lambda$ by the same method used above we find a single eigenvalue, $\lambda=\frac{1}{2}$, as can also be seen from (43). We conclude that the $\lambda_{1}$ branch of the solution of (39) is the proper one since it is the one that gives a finite propagation speed in the limit $\psi \rightarrow \infty$. The $\lambda_{2}$ branch is evidently extraneous.

From the definition of $\lambda$ in (17) with $\lambda$ of order unity we see that

$$
u_{w} \approx a_{e}\left(\frac{\tau_{c}}{\tau_{\text {reac }}}\right)^{1 / 2}
$$

where $a_{e}$ is the electron sound speed. A simple argument can also be used to give this result.* Since $D_{e} \approx a_{e}^{2} \tau_{c}$ a balance between convection and diffusion in the electron conservation equation, (2), gives

$$
u_{v} \approx \frac{a_{e}^{2} \tau_{c}}{L}
$$

with $L$ a length characteristic of the thickness of the wave. A balance between convection and production in (2) gives

$$
\frac{u_{w}}{L} \approx \frac{1}{\tau_{\text {reac }}} .
$$

And eliminating the characteristic length $L$ from (45) and (46) gives (44). A balance between convection, electron diffusion, and production gives the appropriate propagation speed.

\section{WAVE STRUCTURE}

In the limit $\psi \gg 1$ the wave structure can be obtained in analytic form. For arbitrary values of $\psi$ numerical methods must be used. If $\beta \gg \psi \gg 1$ we find from (31), (40) and (42) that

$$
1-H=O\left(\psi^{-1}\right), \quad M-N=O\left(\beta^{-1}\right), \quad G=O\left(\beta^{-1}\right) .
$$

Let us further assume that we may write $\beta=c \psi^{n}$ where $n$ is some appropriate integer larger or equal to unity and $c$ is a constant. For large $\psi$ we assume the following expansion in reciprocal powers of $\psi$,

$$
\begin{aligned}
& N=N_{0}+\psi^{-1} N_{1}, \quad M=N_{0}+\psi^{-1} M_{1} \\
& G=\psi^{-n} G_{n}, \quad H=1+\psi^{-1} H_{1} .
\end{aligned}
$$

If (47) is substituted into (14) we find that to first order

$$
N_{0}=[1+a \exp (2 \lambda \xi)]^{-1 / 2} \text {. }
$$

The constant of integration, $a$, merely determines the location of the wave. This is the same form of solution found by TURCOTTE and ONG (1968). If (47) is substituted into (18) we have to first order

$$
\frac{\mathrm{d} N_{0}}{\mathrm{~d} \xi}+c \lambda N_{0} G_{n}=0 .
$$

* The authors are pleased to acknowledge the referee's suggestion of this argument. 


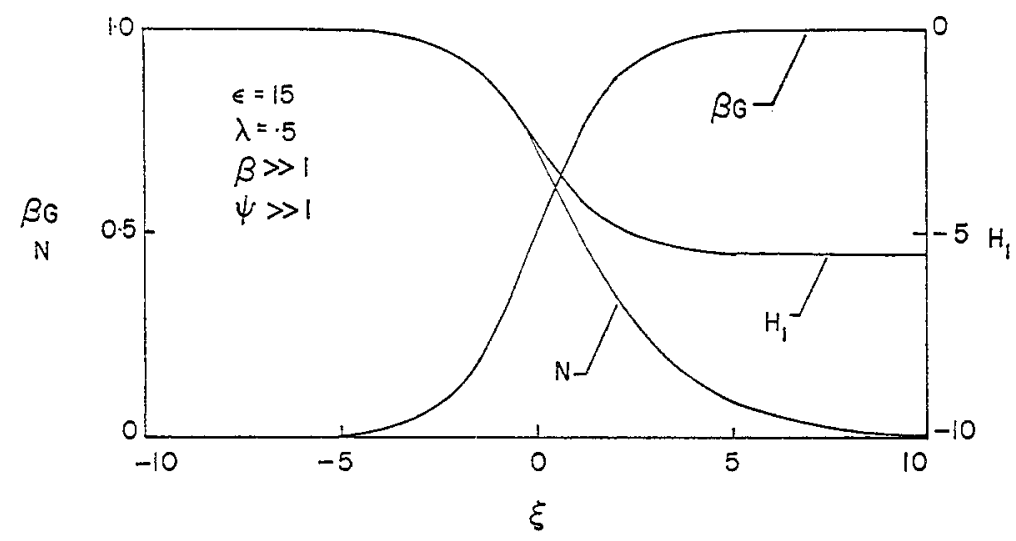

FIG. 2.-Wave structure for $\varepsilon=15$ and $\beta \gg \psi \gg 1$ as given by (52).

Combined with (48) this yields

$$
G_{n}=\frac{a}{c} \mathrm{e}^{2 \lambda \hat{s}}\left(1+a \mathrm{e}^{2 \lambda \hat{s}}\right)^{-1} .
$$

If (47) is substituted into (16) we have to first order

$$
\left(\frac{3}{2}+\varepsilon\right) \frac{\mathrm{d} N_{0}}{\mathrm{~d} \xi}-3 \lambda N_{0} H_{1}=0 .
$$

Using (48) we then obtain

$$
H_{1}=-\left(\frac{\varepsilon}{3}+\frac{1}{2}\right) a \mathrm{e}^{22 \varepsilon}\left(1+a \mathrm{e}^{2 \lambda \varepsilon}\right)^{-1}
$$

When (48), (50) and (51) are substituted into (47) we have

$$
\begin{gathered}
N=\left(1+a \mathrm{e}^{2 \lambda \xi}\right)^{-1 / 2}+O\left(\psi^{-1}\right) \\
M=\left(1+a \mathrm{e}^{2 \lambda \xi}\right)^{-1 / 2}+O\left(\psi^{-1}\right) \\
G=\frac{a}{\beta} \mathrm{e}^{2 \lambda \xi}\left(1+a \mathrm{e}^{2 \lambda \xi}\right)^{-1}+O\left(\beta^{-1} \psi^{-1}\right) \\
H=1-\psi^{-1}\left(\frac{\varepsilon}{3}+\frac{1}{2}\right) a \mathrm{e}^{2 \lambda \xi}\left(1+a \mathrm{e}^{2 \lambda \xi}\right)^{-1}+O\left(\psi^{-2}\right) .
\end{gathered}
$$

If we let $\xi \rightarrow+\infty$ in (52) we find

$$
\begin{gathered}
G_{0}=\beta^{-1}+O\left(\beta^{-1} \psi^{-1}\right) \\
H_{0}=1-\psi^{-1}\left(\frac{\varepsilon}{3}+\frac{1}{2}\right)+O\left(\psi^{-2}\right)
\end{gathered}
$$

which agrees with (40) and (41).

Poisson's equation (15), is not required unless we wish to solve for the detailed structure to $O\left(\psi^{-n}\right)$, i.e. to $O\left(\beta^{-1}\right)$. Since (52) indicates that $\mathrm{d} G / \mathrm{d} \xi \geqslant 0$ everywhere, it follows from (15) that $N \geqslant M$ everywhere. Figure 2 gives a typical solution for $N$, $G$ and $H$ as functions of $\xi$. 


\section{NUMERICAL CALCULATIONS}

A number of restrictive assumptions have been made in the analysis given in this paper. One of the most restrictive is the requirement that neutral-charged particle collisions dominate over collisions between charged particles. However the basic mechanism and the method should be applicable for all degrees of ionization. Therefore we will evaluate the parameters used in the analysis outside the range of validity of the theory.

For atomic hydrogen with $T_{e 0}=10^{4}{ }^{\circ} \mathrm{K}$ we find that $K_{I 0}=5.7 \times 10^{-20} \mathrm{~m}^{3} / \mathrm{sec}$. The ionization potential is $13.6 \mathrm{eV}$, the electron-neutral cross section is $2.3 \times 10^{-19}$ $\mathrm{m}^{2}$, and we assume $n_{n}=10^{21}$ atoms $/ \mathrm{m}^{3}$ with a corresponding electron number density $n_{e 0}=10^{18}$ electrons $/ \mathrm{m}^{3}$. The resulting values for the dimensionless parameters are: $\varepsilon=15.9, \beta=2.5 \times 10^{13}$ and $\psi=1.25 \times 10^{5}$. Clearly both $\beta$ and $\psi$ are very large compared with one and the assumptions made above are valid. The two values for the eigenvalue $\lambda$ are $\lambda_{1}=0.5$ and $\lambda_{2}=1.05 \times 10^{5}$. These correspond to propagation speeds of $36 \mathrm{~m} / \mathrm{sec}$ and $0.25 \mathrm{~m} / \mathrm{sec}$ respectively.

If we consider atomic hydrogen with $T_{e}=10^{5}{ }^{\circ} \mathrm{K}$ and $n_{n}=10^{21}$ atoms $/ \mathrm{m}^{3}$ the equilibrium electron number density is $n_{e 0}=10^{20}$ electrons $/ \mathrm{m}^{3}$ and the lightly ionized assumption is clearly not valid. However the nondimensional parameters can be evaluated and we find that $\varepsilon=1 \cdot 6, \beta \approx 10^{9}$ and $\psi \approx 0 \cdot 4$. The two values of the eigenvalue $\lambda_{\text {are }} \lambda_{1}=1.9$ and $\lambda_{2}=18.6$ and the corresponding values for the propagation speed are $4 \times 10^{4} \mathrm{~m} / \mathrm{sec}$ and $10^{4} \mathrm{~m} / \mathrm{sec}$. At this higher electron temperature the characteristic time for ionization is much shorter and the ionization front can propagate at much higher speeds.

For argon with $T_{e 0}=10^{4}{ }^{\circ} \mathrm{K}$ the electron-neutral cross section is $4 \times 10^{-21} \mathrm{~m}^{2}$ and the ionization potential is $15.8 \mathrm{eV}$. With $n_{n}=10^{21}$ atoms $/ \mathrm{m}^{3}$ the equilibrium electron number density is $n_{e 0}=10^{18}$ electrons $/ \mathrm{m}^{3}$. The values of the dimensionless parameters are $\varepsilon=18 \cdot 4, \beta \approx 10^{15}$ and $\psi=54$. The values of the eigenvalue $\lambda$ are $\lambda_{1}=1 \cdot 10$ and $\lambda_{2}=369$ and the corresponding wave speeds are $185 \mathrm{~m} / \mathrm{sec}$ and $10 \cdot 1$ $\mathrm{m} / \mathrm{sec}$.

\section{APPLICATIONS}

A considerable literature exists on the propagation of potential waves in discharge tubes. If a high voltage is suddenly applied to a discharge tube in the pressure range 0.001-10 torr a discharge is observed to propagate down the tube at speeds up to $10^{7}-10^{8} \mathrm{~m} / \mathrm{sec}$ (BeAMs, 1930; SNOddy et al., 1936, 1937; MitChell and SNODdy, 1947). The propagation speed was a strong function of the applied voltage and the pressure level.

Although no detailed theory has been developed for these waves a qualitative mechanism based on photoionization and a succeeding electron avalanche has been proposed (LOEB, 1965). Under some conditions the theory given in this paper, appropriately modified for geometry and experimental conditions, might be applicable to this problem.

Closely related phenomena are the precursors observed in electric shock tubes. Several investigators (FOWLER and HOOD, 1962; LUBIN and RESLER, 1967) have observed precursor waves travelling at speeds near $10^{6} \mathrm{~cm} / \mathrm{sec}$ ahead of the shock wave. Present theories for these precursor ionization fronts fall mainly into two classes, theories based on electron diffusion and theories based on adsorption of radiation. 
Theories for the upstream diffusion of electrons have been given by PIPKIN (1961, 1963), WeTZEL $(1962,1964)$ and APPLETON (1966). Theories for photo-ionization as the source of the precursor have been given by WeTZEL $(1963,1964)$ and by GERARDO et al. (1963). The theory presented in this paper gives an alternative mechanism for this phenomena. It should be noted, however, that the propagation speed obtained in this paper is a strong function of the electron temperature because of the exponential character of the ionization rate. Therefore the electron temperature must be known with considerable accuracy before a direct comparison of theory and experiment can be made.

\section{CONCLUSIONS}

In this paper a new wave propagation mechanism has been investigated. It is found that an ionization front may propagate into a nonionized gas at a well defined speed. Two propagation speeds have been found, see (39), as the solution of a nonlinear eigenvalue problem. One of the solutions is rejected on the basis of a limiting solution. The method and results are similar to those used to determine the propagation of laminar flames.

Although the results obtained here are restricted to a lightly ionized gas obeying a particular ionization reaction, the method can easily be extended to higher degrees of ionization and more complicated ionization reactions. The results could also be extended to include the effects of applied magnetic fields and might be applicable to ionizing shock waves.

Acknowledgment-This work was supported by the U.S. Air Force Office of Scientific Research under Grant AF-AFOSR-825-67A (RM and RSBO) and Contract AF 49(638)-1346 (DLT).

\section{REFERENCES}

Appleton J. P. (1960) Physics Fluids 9, 336.

Beams J. W. (1930) Phys. Rev. 36, 997.

Burgers J. M. (1960) Plasma Dynamics (F. H. Clausser, Ed.), Addison-Wesley, Reading, Mass.

Fowler R. G. and Hood J. D. (1962) Phys. Rev. 128, 991.

Gerardo J. B., Hendricks C. D. and Goldstern L. (1963) Physics Fluids 6, 1222.

LOED L. B. (1965) Science 148, 1417.

LuBIN M. J. and RESLER E. L. (1967) Physics Fluids 10, 1.

MrtChell F. H. and SNODdY L. B. (1947) Phys. Rev. 72, 1202.

PIPKIN A. C. (1961) Physics Fluids 4, 1298.

Prpkin A. C. (1963) Physics Fluids 6, 1382.

SNoddy L. B., Beams J. W. and Dietrich J. R. (1936) Physs. Rev. 50̂, 469.

SNoddy L. B., Dietrich J. R. and BeAMS J. W. (1937) Phys. Rev. 52, 739.

TuRCoTte D. L. and ONG R. S. B. (1968) J. Plasma Phys. 2, 145.

WeTZei L. (1962) Physics Fluids 5, 827.

Wetzel L. (1963) Physics Fluids 6, 750.

Wetzel L. (1964) AIAA J. 2, 1208. 